\title{
Bidding strategy of power generation companies in the centralized electricity spot market
}

\author{
Jinshan Han ${ }^{1}$, Pengfei $\mathrm{Li}^{1,}{ }^{*}$, Tingting Zhang ${ }^{1}$, Lingxiang Wang ${ }^{1}$ and Dunnan Liu ${ }^{1}$ \\ ${ }^{1}$ North China Electric Power University, Beijing, 102206, China
}

\begin{abstract}
With the development of China's power market reform, the spot market has gradually opened up. The spot market is characterized by volatility and uncertainty. Generators urgently need to manage spot market trading income and risks to avoid the risk of spot price fluctuations. This paper studies the bidding strategies of power generation companies in the concentrated spot market, proposes a risk-return coordination method based on the probability of winning bids and conducts case verification. The example shows that the method can assist the power generation companies to formulate bidding strategies.
\end{abstract}

\section{Introduction}

In August 2017, the National Development and Reform Commission and the State Energy Administration issued a Notice on the pilot work on the Construction of the Spot Market for Electricity, which identified eight regions (Guangdong, Mengxi, Zhejiang, Shanxi, Shandong, Fujian, Sichuan and Gansu) as the first pilots.

The rules published in the pilot areas have made it clear in many places that the medium- and long-term contract market adopts the CFD model and settles the difference based on the current spot market price. Considering the volatility and uncertainty of spot market prices, generators urgently need to manage spot market transaction revenue and risks, so as to avoid spot price fluctuation risks and achieve good control of their own risks.

In the research of trading risk in the power market, there have been relatively many studies. In the study of measuring risk based on cost variance, literature [1] studied the bidding strategy of power generation companies based on the method of measuring risk based on profit variance. Reference [2] used the method of cost variance to construct a power purchase strategy model with minimum power purchase costs. Literature [3] measured the power purchase risk of power supply companies in the long-term market and the short-term market by income variance. In recent years, $\mathrm{VaR}$ and $\mathrm{CVaR}$, the risk measurement methods in the financial field, have been increasingly used in the power market risk measurement [4-8], mostly as a risk measurement method. At present, there are many researches based on $\mathrm{VaR}$ and $\mathrm{CVaR}$ to measure the transaction risk of power purchase side [9-10].

This paper studies the bidding strategies of power generation companies in the spot mode. First of all, it sorted out China's electricity market mechanism, then analyzed the trading needs of power generation companies in the spot market, and then based on the winning probability curve, proposed a bidding strategy for power companies in the spot market. Finally, it is verified based on a calculation example.

\section{Electricity spot market principle}

The spot market refers to the market of electricity products trading in the near future and in a shorter period of time. It requires the consideration of supply and demand balance and security constraints when organizing market transactions. All medium- and longterm bilateral energy transactions need to be traded on the spot market, and the next day's power generation schedules are formed on the premise of ensuring safety. Spot markets include day-ahead markets, intra-day markets, real-time markets, and ancillary services markets.

The spot market is generally divided into two kinds of decentralized and centralized market by market form:

(1) Decentralized: based on medium- and long-term physical contracts, the parties in the previous stage to determine their own daily electricity curve, real-time deviation of electricity through real-time balance trading adjustment, the implementation of part of the power bidding mechanism. Its essence is that the power generation party and the purchaser shall make selfdispatching self-arrangement according to the bilateral contract signed, and the system dispatching institution shall do its best to ensure the execution of the contract and be responsible for the balanced dispatch of electricity. China's decentralized spot market pilot for Sichuan, Fujian.

(2) Centralized: to the medium- and long-term differential contract management market risk, with spot trading using the full-charge centralized bidding of the power market model. Its essence is to determine the unit combination and power generation curve based on safety

* Corresponding author: 2210055766@qq.com 
constraints, and it is a trading model which is closely related to the operation of the power grid and optimizes all kinds of transactions. China's centralized spot market pilot for Guangdong, Zhejiang.

\section{Request for quotations from power generation companies in the centralized spot market}

The centralized power market mainly manages market risks with medium- and long-term price difference contracts, and cooperates with spot transactions to adopt the power market model of centralized bidding for full power. The centralized power market is mainly based on the signing of price difference contracts, and the resource allocation efficiency is higher, but the market rules are more complicated and it is difficult to monitor market power. In the centralized power market, the next day's power generation plan is determined by the power generation enterprise, the user and the electricity sales entity through spot market bidding to determine the total power generation and power consumption curve for the next day. The auction is conducted with a period of 5-15 minutes. The auction mode is full power auction. The optimization result is the power generation curve, settlement price, auxiliary service capacity, auxiliary service price, etc. in the auction cycle.

When participating in the full power bidding in the centralized spot market, power generation enterprises should use the advantages of power generation capacity, reasonably declare the power-electricity price curve, and use part of the capacity to pursue the marginal transaction price to increase the clearing price and increase the electricity sales revenue.

\section{Bidding Decision of Power Generation Companies in Centralized Spot Market}

(1) Optimization of the bidding strategy for the highest capacity segment

The role of the highest capacity segment of the power generation company is to significantly increase the market price in the case of insufficient market supply, and the other is to chase the market's marginal electricity price, so that the power generation company can use more power generation capacity and obtain higher price. In the case that the market may have insufficient reserve capacity, the quotation of the highest capacity segment of the power generation company shall follow the market's marginal price.

In the more mature electricity spot market, bidding strategies of power generation companies are also relatively stable. In the case of little change in the market environment, the market transactions in the spot electricity market for several consecutive days should be similar--in the same period, the market price and generation load obtained by each power generation company are basically similar. This allows power generation companies to optimize bids based on market announcements a few days before the transaction.
In the actual electricity spot market, the quotation of the power generation company is a power-electricity price curve. The quotation curve is segmented and stepped. The power generation company can only use a limited number of capacity segments. The curve is shown in Figure 1. The diagonal line in the figure represents the market's demand line, and its intersection is the market price. When quoting, if the market's marginal electricity price is higher than the minimum capacity cost of the power generation company, the power generation company should make the price of the highest capacity segment chase the market's marginal electricity price.

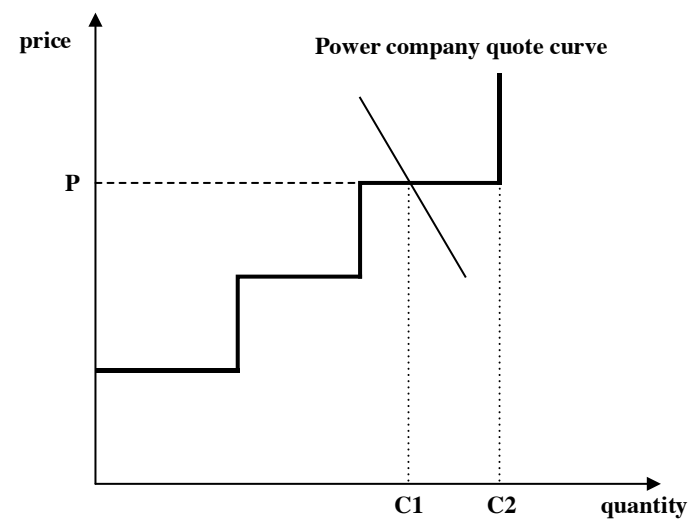

Fig. 1. market quote curve

If the supply capacity of the market marginal price $(\mathrm{P})$ is greater than the power demand $(\mathrm{C} 1)$, a portion of the power generation capacity quoted at the marginal price $(\mathrm{C} 2-\mathrm{Cl})$ cannot be used and can be called the same price spare capacity, and the power generation capacity at the marginal price is allocated to the relevant power generation companies on a rotational basis, if the same price spare capacity is large, the load of each related power generation company changes frequently in the case of system load and market conditions. At this time, if the same period of the previous day in the market spare capacity is more, the power generation company in this period of the highest capacity segment of the quotation should be reduced, if the spare power generation capacity is small, the power generation company quote the highest capacity segment price should be increased.

(2) Trading decision method: risk-reward coordination method

The risk return curve is shown in Fig 2.

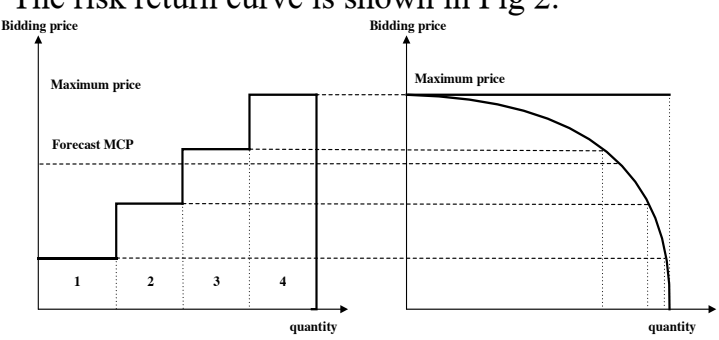

Fig. 2. risk return curve

Step 1: Predict the market marginal electricity price $\mathrm{MCP}$ 
Step 2: Give the probability distribution curve of the winning bid (based on the previous quote and the result of the transaction statistics);

Step 3: Determine the required electricity;

Step 4: Set the probability of winning the bid for paragraphs 2, 3, and 4 and the corresponding electricity price based on risk appetite.

Among them, the winning probability distribution curve can be drawn based on historical data. The clearing price of each transaction is analyzed. The probability of winning bids for all clearing price intervals is $100 \%$, and the probability of winning bids above the trading price range is 0 . The cumulative average value is obtained according to the results of each transaction to obtain the winning probability distribution curve.
Different bidding segments correspond to different bid winning probabilities, so that the expected return value can be calculated, and different bidding intervals can be set according to the requirements of risk standards.

\section{Example analysis}

This trading decision method based on the winning probability curve requires a large amount of historical data and is more adaptable in the spot market. It is also applicable to the decentralized power market when applied to the spot market's bidding strategy. Table 1 shows the price data of 20-day clearing prices in an electricity market.

Table 1. Market clearing price

\begin{tabular}{|c|c|c|c|c|c|c|c|c|}
\hline $\begin{array}{c}\text { Marginal quote } \\
(\mathrm{RMB} / \mathrm{kWh})\end{array}$ & 0.14 & 0.15 & 0.16 & $\ldots$ & 0.28 & 0.29 & 0.3 & 0.31 \\
\hline 0.2932 & 1 & 1 & 1 & $\ldots$ & 1 & 0 & 0 & 0 \\
\hline 0.2913 & 1 & 1 & 1 & $\ldots$ & 1 & 0 & 0 & 0 \\
\hline 0.3183 & 1 & 1 & 1 & $\ldots$ & 1 & 1 & 1 & 0 \\
\hline 0.2933 & 1 & 1 & 1 & $\ldots$ & 1 & 0 & 0 & 0 \\
\hline 0.3195 & 1 & 1 & 1 & $\ldots$ & 1 & 1 & 1 & 0 \\
\hline 0.2981 & 1 & 1 & 1 & $\ldots$ & 1 & 0 & 0 & 0 \\
\hline 0.2163 & 1 & 1 & 1 & $\ldots$ & 0 & 0 & 0 & 0 \\
\hline 0.1538 & 1 & 0 & 0 & $\ldots$ & 0 & 0 & 0 & 0 \\
\hline 0.2192 & 1 & 1 & 1 & $\ldots$ & 0 & 0 & 0 & 0 \\
\hline 0.2705 & 1 & 1 & 1 & $\ldots$ & 0 & 0 & 0 & 0 \\
\hline 0.2711 & 1 & 1 & 1 & $\ldots$ & 0 & 0 & 0 & 0 \\
\hline 0.3068 & 1 & 1 & 1 & $\ldots$ & 1 & 1 & 0 & 0 \\
\hline 0.2762 & 1 & 1 & 1 & $\ldots$ & 0 & 0 & 0 & 0 \\
\hline 0.2810 & 1 & 1 & 1 & $\ldots$ & 0 & 0 & 0 & 0 \\
\hline 0.2610 & 1 & 1 & 1 & $\ldots$ & 0 & 0 & 0 & 0 \\
\hline 0.2645 & 1 & 1 & 1 & $\ldots$ & 0 & 0 & 0 & 0 \\
\hline 0.2823 & 1 & 1 & 1 & $\ldots$ & 0 & 0 & 0 & 0 \\
\hline 0.3136 & 1 & 1 & 1 & $\ldots$ & 1 & 1 & 1 & 0 \\
\hline 0.2253 & 1 & 1 & 1 & $\ldots$ & 0 & 0 & 0 & 0 \\
\hline 0.2381 & 1 & 0.95 & 0.95 & $\ldots$ & 0.4 & 0.2 & 0.15 & 0 \\
\hline AVERAGE & & 1 & 1 & $\ldots$ & 0 & 0 & 0 & 0 \\
\hline
\end{tabular}

The winning probability curve is obtained by processing the data, as shown in Figure 3. The fitting resulting secondary function curve is $y=-0.1836 \mathrm{x}^{2}$ $0.037 \mathrm{x}+0.3058$.

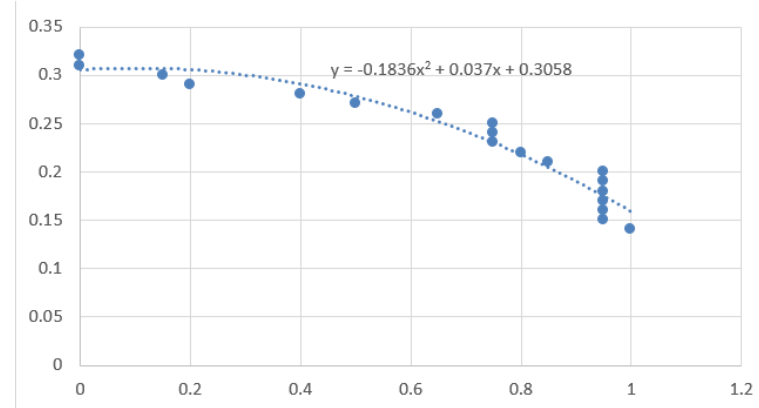

Fig. 3. winning probability curve
The battery can be allocated according to your own risk appetite. Develop different segmented quote strategies, and then predict the returns of each quote strategy. This allows you to choose the best-thanexpected quote scenario.

$$
E=\sum_{n=1}^{N} Q_{i} * p_{i} * P_{i}
$$

In the formula, $\mathrm{E}$ is the expected return, $\mathrm{n}$ is the number of quoted segments, the i-th power segment, the corresponding quote, the i-th quoted settlement price, and the $\mathrm{i}$-th quoted bid rate.

If the market adopts PAB settlement method, $p_{i}^{\prime}$ will be the price quoted in paragraph $\mathrm{P}_{\mathrm{i}}$.

If the market adopts the MCP calculation method, when all $\mathrm{P}_{\mathrm{i}}$ are less than the predicted MCP, the MCP is $p_{i}^{\prime}$ and the winning bid rate is corrected to 1 ; 
When $\mathrm{Pi}$ is greater than or equal to $\mathrm{MCP}$, the marginal price of the market increases based on the probability of winning the bid, and is set to the highest transaction price, that is, all $\mathrm{Pi}$ 's take the maximum of $\mathrm{Pi}$; The winning rate of $\mathrm{Pi}$ exceeding or equal to the MCP part is calculated according to the winning probability curve.

\section{Conclusions}

This paper studies the bidding strategies of power generation companies in the centralized spot market, and constructs a trading decision method based on the probability curve of winning bids, which has certain practicability. The trading decision method based on the winning probability curve has a large demand for historical data and is relatively adaptable in the spot market. When applied to the spot market's quotation strategy, it is also applicable to the decentralized power market.

\section{Acknowledgements}

Science and Technology Project of State Grid Corporation of China, Integrated Operation Control Strategy of Cross District Whole Network Adapting to the Development of New Energy, Number: SGTYHT/16-JS-198.

\section{References}

1. X.S. Ma, J.X. Liu, F.L. Wen, et al. Journal of north China electric power university, Research on bidding strategy of power generation companies considering risk and CFD,32, 37(2005)

2. Conejo A J, Carrion M. IEEE ProceedingsGeneration, Transmission and Distribution, Riskconstrained electricity procurement for a large consumer, 153, 407(2006)

3. J Guo, W Jiang, Z.F. Tan. Power grid technology, Study on optimal power purchase of power supply companies under risk conditions,28,18(2004)

4. Z. Zhou, J.W. Kang, J.H. Chen, et al. Chinese journal of electrical engineering, Application of monte carlo method in short-term financial risk assessment of power market,24,74(2004)

5. Denton M, Palmer A, Masiello R, et al. IEEE Trans on Power Systems, Managing Market Risk in Energy, 18, 494(2003)

6. BjRkqvist O, Idefeldt J, Larsson A. Energy Policy, Risk assessment of new pricing strategies in the district heating market: A case study at Sundsvall Energy $A B, \mathbf{3 8}, 2171(2010)$

7. Yoshida Y. IFSA/NAFIPS, Optimization of valueat-risk portfolios in uncertain lognormal models (2013) thi

8. Krzemienowski A, Szymczyk S. Annals of Operations Research, Portfolio optimization with a copula-based extension of conditional value-atrisk,237,219(2016)

9. Y.A. Zheng, G.Y. Li, M Zhou. Chinese journal of electrical engineering, Research on fuzzy optimization of power purchase combination strategy for large users,30,98(2010)

10. M Zhou, Y.L. Nie, G.Y. Li, et al. Chinese journal of electrical engineering, Long-term power purchase scheme and risk assessment in power market,26,116(2006) 